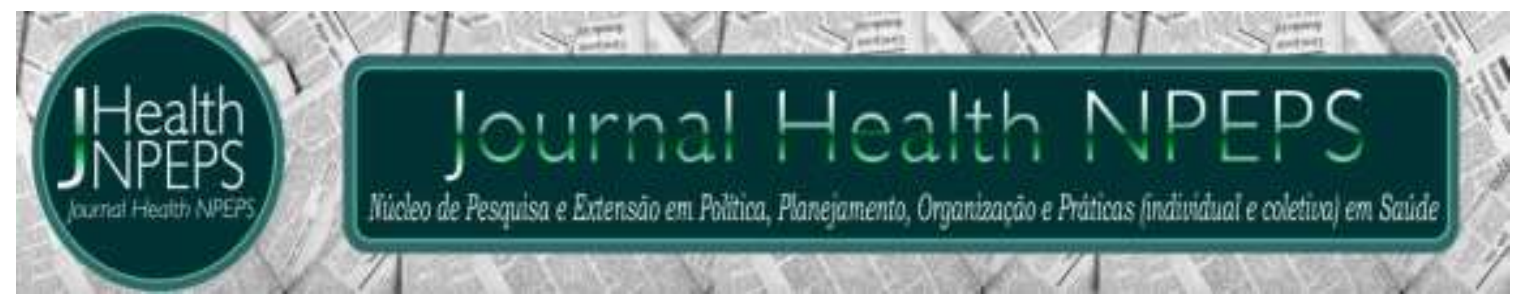

http://dx.doi.org/10.30681/252610104575

ARTIGO DE REVISÃO

\title{
Diagnósticos de enfermagem para pacientes com COVID-19
}

\author{
Nursing diagnoses for patients with COVID-19
}

Diagnostico de enfermeria para pacientes con COVID-19

\section{Tays Pires Dantas ${ }^{1}$, Cosmo Alexandro da Silva de Aguiar², Vithória Régia Teixeira Rodrigues $^{3}$, Raul Roriston Gomes da Silva ${ }^{4}$, Maria Isabel Caetano da Silva ${ }^{5}$, Luis Rafael Leite Sampaio ${ }^{6}$, Woneska Rodrigues Pinheiro ${ }^{7}$}

\begin{abstract}
RESUMO
Objetivo: elencar com base nas manifestações clínicas da doença, os principais diagnósticos de enfermagem que podem ser aplicados para crianças, adultos, gestantes e idosos com COVID-19. Método: revisão integrativa da literatura nacional e internacional, realizada no período de março a maio de 2020 , a partir do cruzamento entre os termos: "pacientes", "infecções por coronavírus" e "sinais e sintomas", usando o operador booleano and. Selecionou-se 26 estudos, apresentando em quadro. Utilizouse a taxonomia da Associação Norte-Americana de Diagnósticos de Enfermagem e a Classificação Internacional para a Prática de Enfermagem para elencar os diagnósticos
\end{abstract}

\footnotetext{
${ }^{1}$ Acadêmica do 8o semestre do curso de graduação em Enfermagem. Bolsista institucional do programa de extensão Ambulatório de Enfermagem em Estomaterapia. Universidade Regional do Cariri (URCA), Departamento de Enfermagem. Juazeiro do Norte, Ceará, Brasil. E-mail: tayspires12@gmail.com ORCID ID: https://orcid.org/0000-0003$\underline{0374-3865}$

${ }^{2}$ Acadêmico do 8o semestre do curso de graduação em Enfermagem. Universidade Regional do Cariri (URCA), Departamento de Enfermagem. Crato, Ceará, Brasil. E-mail: cosmoaguiar84@gmail.com ORCID ID: https://orcid.org/0000-0001-5311-6814

${ }^{3}$ Acadêmica do 8o semestre do curso de graduação em Enfermagem. Bolsista institucional BPI- Funcap. Universidade Regional do Cariri (URCA), Departamento de Enfermagem. Crato, Ceará, Brasil. E-mail: vithoriaregia00@gmail.com ORCID ID: https://orcid.org/0000-0003-3253-9134

${ }^{4}$ Acadêmico do 8o semestre do curso de graduação em Enfermagem. Técnico em Enfermagem Emergencista no Hospital Regional do Cariri (HRC). Universidade Regional do Cariri (URCA), Departamento de Enfermagem. Juazeiro do Norte, Ceará, Brasil. E-mail: rroriston@live.com ORCID ID: https://orcid.org/0000-0002-8576-5875

${ }^{5}$ Acadêmica do 9o semestre do curso de graduação em Enfermagem. Universidade Regional do Cariri (URCA), Departamento de Enfermagem. Crato, Ceará, Brasil. E-mail: mariaisabelcs28@outlook.com ORCID ID: https://orcid.org/0000-0001-5389-3664

${ }^{6}$ Enfermeiro. Estomaterapeuta TISOBEST. Doutor em Farmacologia. Docente Adjunto da Universidade Regional do Cariri (URCA). Crato, Ceará, Brasil. E-mail: rafael.sampaio@urca.br ORCID ID: https://orcid.org/0000-0003-1437-9421 Autor principal - Endereço para correspondência: Rua Cel. Antônio Luis, 1161 - 63105-000 - Pimenta - Crato/CE, Brasil.

${ }^{7}$ Enfermeira. Doutora em Ciências da Saúde. Docente Adjunto da Universidade Regional do Cariri (URCA). Juazeiro do Norte, Ceará, Brasil. E-mail: woneskar@gmail.com ORCID ID: https://orcid.org/0000-0003-3353-9240
}

Este artigo está licenciado sob forma de uma licença Creative Commons Atribuição 4.0 Internacional, que permite uso irrestrito, distribuição e reprodução em qualquer meio, desde que a publicação original seja corretamente citada. 
pertinentes ao quadro clínico frequentemente presente entre participantes com covid19. Resultados: conforme os sinais e sintomas presentes na fase aguda da doença em pacientes sob internação e em uso de terapia medicamentosa, os diagnósticos predominantes foram proteção ineficaz relacionado à incapacidade de proteção contra agente infeccioso evidenciado por tosse, calafrios e fadiga, presença de tosse, Hipertermia relacionada a processo infeccioso evidenciado por pele quente ao toque, padrão respiratório ineficaz relacionado à dor e fadiga evidenciado por dispneia. Considerações finais: alguns diagnósticos são prevalentes, entretanto, a avaliação deve ser individual, bem como a assistência, baseada nas necessidades de cada indivíduo.

Descritores: Pacientes; Infecções por Coronavírus; Sinais e Sintomas.

\section{ABSTRACT}

Objective: to list, based on the clinical manifestations of the disease, the main nursing diagnoses that can be applied to children, adults, pregnant women and the elderly with COVID-19. Method: integrative review of national and international literature, carried out from March to May 2020, based on the crossing between the terms: "patients", "coronavirus infections" and "signs and symptoms" , using the Boolean operator and. 26 studies were selected and presented in a table. The taxonomy of the North American Association of Nursing Diagnoses and the International Classification for Nursing Practice were used to list the diagnoses relevant to the clinical picture frequently present among participants with covid-19. Results: according to the signs and symptoms present in the acute phase of the disease in patients hospitalized and using drug therapy, the prevalent diagnoses were ineffective protection related to the inability to protect against an infectious agent evidenced by cough, chills and fatigue, presence of cough, related hyperthermia an infectious process evidenced by hot skin to the touch, ineffective breathing pattern related to pain and fatigue evidenced by dyspnea. Final considerations: some diagnoses are prevalent, however, the assessment must be individual, as well as assistance, based on the needs of each individual.

Descriptors: Patients; Coronavirus Infections; Signs and Symptoms.

\section{RESUMEN}

Objetivo: enumerar, con base en las manifestaciones clínicas de la enfermedad, los principales diagnósticos de enfermería que pueden aplicarse a niños, adultos, mujeres embarazadas y ancianos con COVID-19. Método: revisión integradora de la literatura nacional e internacional, realizada entre marzo y mayo de 2020, basada en el cruce entre los términos: "pacientes", "infecciones por coronavirus" y "signos y síntomas" , utilizando el operador booleano y. Se seleccionaron 26 estudios y se presentaron en una tabla. La taxonomía de la Asociación Norteamericana de Diagnósticos de Enfermería y la Clasificación Internacional para la Práctica de Enfermería se utilizaron para enumerar los diagnósticos relevantes para el cuadro clínico frecuentemente presente entre los participantes con covid-19. Resultados: de acuerdo con los signos y síntomas presentes en la fase aguda de la enfermedad en pacientes hospitalizados y que usan terapia farmacológica, los diagnósticos prevalentes fueron protección ineficaz relacionada con la incapacidad de protección contra un agente infeccioso evidenciado por tos, escalofríos y fatiga, presencia de tos, hipertermia relacionada un proceso infeccioso evidenciado por la piel caliente al tacto, un patrón de respiración ineficaz relacionado con el dolor y la fatiga evidenciada por la disnea. Consideraciones finales: algunos diagnósticos son frecuentes, sin embargo, la evaluación debe ser individual, así como asistencia, según las necesidades de cada individuo.

Descriptores: Pacientes; Infecciones por Coronavirus; Signos y Síntomas. 
INTRODUÇÃO

Os coronavírus constituem um grupo de vírus que são capazes de causar infecções respiratórias, com repercussões clínicas que variam de leves até letais. A primeira detecção desse tipo de vírus em humanos foi em 1937, entretanto, somente em 1965 determinou como sendo coronavírus; graças ao seu perfil microscópico semelhante a uma coroa. Em dezembro de 2019, após casos registrados em Wuhan, província de Hubei, na China, foi descoberto um novo tipo de coronavírus, sendo denominado de SARS-CoV-2, causador da doença Coronavirus Disease 2019 (COVID-19) 1.

Com disseminação da COVID-19 para todos os continentes, em um curto período de tempo esta se tornou uma pandemia. Em abril de 2020 já havia mais de 2,5 milhões de infectados em todo o mundo, no Brasil, até o dia 25 de abril, o número já ultrapassava os 50.000 casos $^{1}$. Atualmente, o cenário é de incertezas e desafios, ainda pouco se sabe sobre a doença e quais suas consequências a longo prazo.

Normalmente, o quadro clínico apresentado pelo paciente é similar à síndrome gripal, com tosse, febre, dor no corpo, e por vezes dor de cabeça e/ou diarreia. Entretanto, no agravamento do caso, o indivíduo passa a sofrer complicações principalmente pulmonares, ocasionando dificuldade respiratória ${ }^{2}$. A taxa de letalidade é variável, na China, por exemplo, em abril de 2020, a taxa era de 3\%; no Brasil, no mesmo período, a taxa alcançava $6,3 \%^{3}$.

Diante do exposto, faz-se necessário uma assistência clínica multidisciplinar especializada para esses pacientes, tendo em vista as nuances no quadro clínico que apresentam. Entretanto, destaca-se a importância do profissional enfermeiro, visto que este desempenha papel de liderança nos serviços de saúde ${ }^{4}$ promovendo direcionamento de ações ao coordenar principalmente a equipe de enfermagem. Nesse sentido, a Sistematização da Assistência de Enfermagem (SAE) é uma importante aliada do enfermeiro, sendo fundamental na prática clínica assistencial e essencial para o processo de trabalho de toda a equipe de enfermagem.

Um dos caminhos utilizados para sistematizar a assistência de enfermagem nos serviços de saúde é o Processo de Enfermagem, método constituído por: 1) histórico de 
enfermagem, 2) diagnóstico de enfermagem, 3) plano assistencial, 4) plano de cuidados, e 5) evolução de enfermagem ${ }^{5}$. Entretanto, destaca-se a etapa 2; esse é o momento base para a seleção das ações ou intervenções com as quais se objetiva alcançar os resultados esperados ${ }^{4,5}$. Traçar Diagnósticos de Enfermagem (DE) em pacientes com COVID-19 é fundamental para o alcance de um cuidado holístico, voltado para todas as necessidades humanas. Ressalta-se que os diagnósticos devem ser constantemente atualizados, a cada nova avaliação, uma vez que o estado de saúde de pacientes com a COVID-19 não é estável, favorecendo mudanças nas necessidades desses indivíduos.

Diante disso, o objetivo do estudo consiste em elencar com base nas manifestações clínicas da doença, os principais diagnósticos de enfermagem que podem ser aplicados para pacientes com COVID-19, segundo a taxonomia da Associação Norte-Americana de Diagnósticos de Enfermagem (NANDA-I) versão 2018-20206, e a Classificação Internacional para a Prática de Enfermagem (CIPE) versão 20197.

\section{MÉTODO}

Trata-se de uma revisão integrativa da literatura que seguiu as etapas propostas por Mendes, Silveira e Galvão ${ }^{8}$, de maneira organizada e sistematizada, permitindo os pesquisadores explorar o tema estudado a partir da elaboração da pergunta de pesquisa, busca e seleção dos estudos, extração dos dados, avaliação dos estudos, síntese dos resultados e apresentação da revisão. A pesquisa ocorreu nos meses de março a maio de 2020. Inicialmente houve a indagação: "Quais os diagnósticos de enfermagem podem ser propostos às pessoas com COVID-19?".

Em seguida foram escolhidos os Descritores em Ciências da Saúde (DeCS): Pacientes, Infecções por Coronavírus, Sinais e Sintomas; e seus respectivos Medical Subject Headings (MeSH): Patients, Coronavirus infections e Signs and Symptoms.

Após definição dos descritores, a busca foi realizada em pares por dois revisores de forma independente no portal da Biblioteca Virtual em Saúde (BVS). Utilizou-se o método de busca avançada, e como estratégia de investigação o cruzamento entre os termos por meio do operador booleano AND: Pacientes AND Infecções por Coronavírus AND Sinais e Sintomas; 
Patients AND Coronavirus infections AND Signs and Symptoms; que resultou em 333 publicações.

Logo, aplicaram-se os filtros: texto completo disponível; gratuito; tipo de documento artigo, sem restrições de ano de publicação e idioma, para que todos as pesquisas relacionadas a temática fossem avaliadas. 0 processo de filtragem resultou em estudos distribuídos nas bases de dados: Literatura Latino-Americana e do Caribe em Ciências da Saúde (LILACS), e Medical Literature Analysis and Retrieval System Online (MEDLINE), os quais passaram pela leitura de título e resumo, verificando sua adequação à temática em estudo. Como critérios de inclusão, utilizou-se: relatos de casos que apontem manifestações clínicas em diferentes amostras de pacientes infectados com COVID-19 nos grupos: crianças, adultos, gestantes e idosos. E foram excluídos os artigos repetidos, manuscritos pré-aprovados apenas com a versão pré-print disponibilizada na página da revista e estudos de casos cujos pacientes estivessem apresentando quadro clínico assintomático.

0 processo de seleção dos artigos foi exposto com detalhes através do instrumento Preferred Reporting Items for Systematic Review and MetaAnalyses (PRISMA) ${ }^{9}$, fluxograma que melhora a demonstração do relato da revisão (Figura 1).

Figura 1 - Fluxo de seleção dos estudos com as fases de uma revisão integrativa pelo PRISMA. Crato CE, Brasil, 2020.
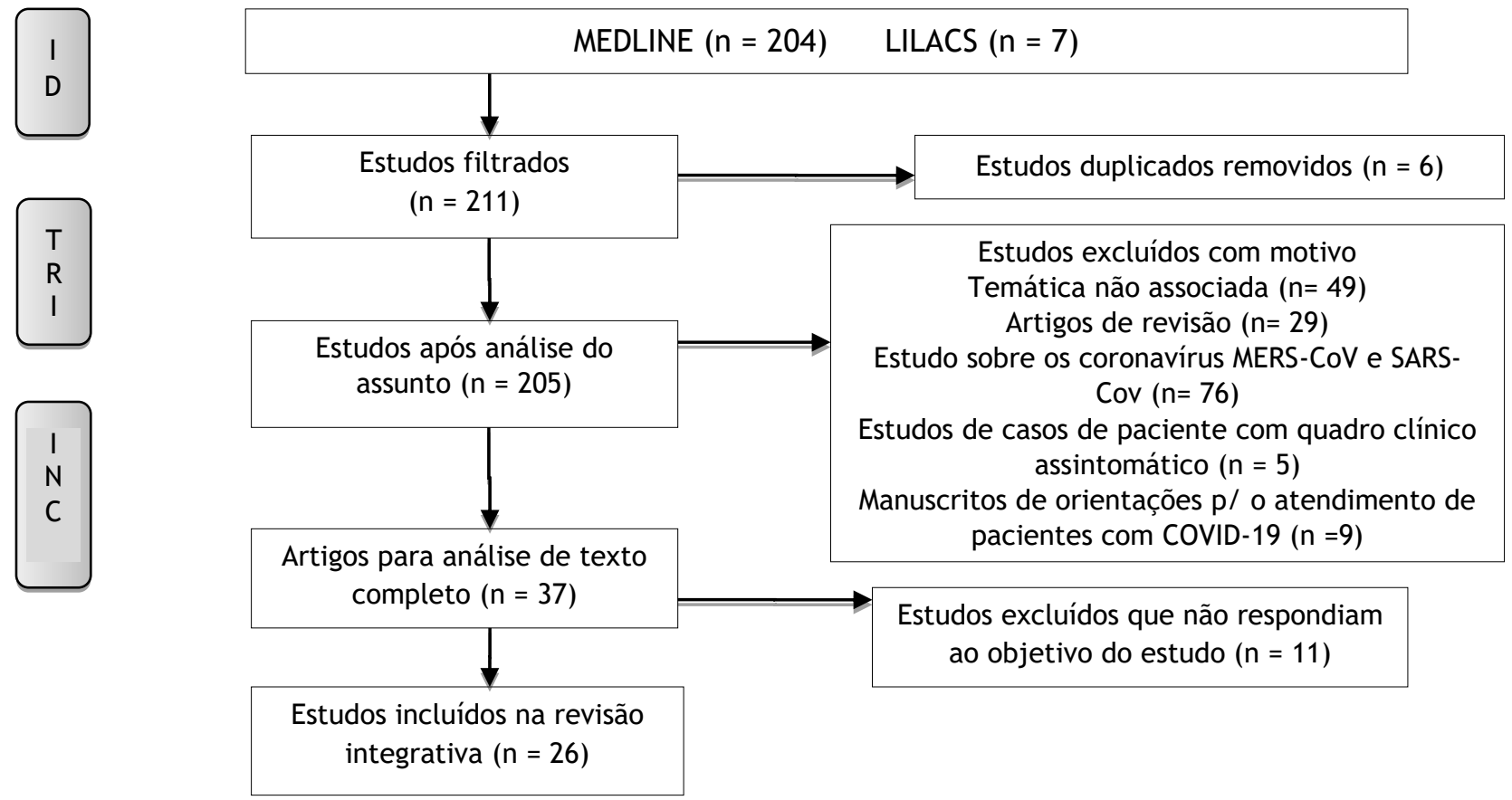
Durante a coleta de dados foi elaborado um quadro composto pelas informações dos artigos selecionados, a saber: título, autores, ano de publicação, objetivo e amostra (Quadro 1 - Anexo). Em seguida, foram agrupados os dados relatados nas publicações e a partir da extração de todos os sinais e sintomas verbalizados pelos pacientes no momento da admissão no serviço hospitalar e as manifestações clínicas observadas pela equipe de saúde durante o acompanhamento e avaliação física dos casos de COVID-19, tanto em pacientes internados nas enfermarias, como em UTI, os autores elencaram os DE que podem ser aplicados a esse público, tendo como base cientifica estudos disponíveis sobre a temática e as taxonomias: NANDA-I e CIPE.

Os achados foram analisados à luz da literatura pertinente, conciliando as publicações da Organização Mundial da Saúde (OMS), Ministério da Saúde (MS) e de artigos desenvolvidos sobre o tema em questão.

\section{RESULTADOS}

A partir da leitura dos estudos disponíveis na literatura mundial sobre casos de pessoas com a infecção por SARS-Cov-2, foram selecionados 26 relatos de casos, os quais passaram por uma análise detalhada e verificação dos sinais e sintomas frequentemente apresentados entre os indivíduos com COVID-19. Notou-se prevalência do quadro clínico composto por

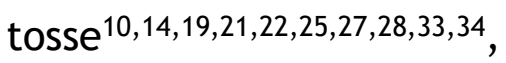
hipertermia $11,14,16,17,20-23,27,32$, dor aguda $12,14,18,21,23$, vômito $12,15,18,26,30$, diarreia ${ }^{12,15,22,27,31}$ e dispneia ${ }^{10,14,19,20,35}$. Entretanto, além destes, também estiveram presentes sinais como edema em membros inferiores ${ }^{13}$, hemoptise ${ }^{29,33}$, dor no peito e palpitações ${ }^{12,13,17,22,25,26}$ (Quadro 2).

\begin{tabular}{l} 
Quadro 2 - Sinais e sintomas apresentados por pacientes com COVID-19, e \\
população predominante com este quadro clínico. Crato-CE, Brasil, 2020. \\
\begin{tabular}{|c|c|}
\hline POPULAÇÃO & SINAIS E SINTOMAS \\
\hline Crianças & Dor de garganta \\
& Coriza \\
& Hipertermia \\
& Expectoração \\
& Fadiga \\
& Vômitos e diarreia \\
Dispneia \\
Calafrios
\end{tabular} \\
\hline
\end{tabular}




\begin{tabular}{|c|c|}
\hline Adultos & $\begin{array}{c}\text { Edema em membros inferiores } \\
\text { Tontura } \\
\text { Dor abdominal } \\
\text { Palpitações e aperto no peito } \\
\text { Cefaleia } \\
\text { Coriza } \\
\text { Dor no peito } \\
\text { Náusea } \\
\text { Dor de garganta } \\
\text { Dispneia } \\
\text { Vômito e diarreia } \\
\text { Calafrios } \\
\text { Fadiga } \\
\text { Expectoração produtiva } \\
\text { Mialgia } \\
\text { Hipertermia } \\
\text { Tosse }\end{array}$ \\
\hline Gestantes & $\begin{array}{c}\text { Mal-estar } \\
\text { Insônia, depressão e estresse } \\
\text { Dor no peito } \\
\text { Cefaleia } \\
\text { Vômito e diarreia } \\
\text { Tosse } \\
\text { Expectoração produtiva } \\
\text { Calafrios } \\
\text { Hipertermia } \\
\text { Mialgia } \\
\text { Dispneia }\end{array}$ \\
\hline Idosos & $\begin{array}{l}\text { Hemoptise } \\
\text { Fadiga } \\
\text { Mialgia } \\
\text { Tosse } \\
\text { Hipertermia }\end{array}$ \\
\hline
\end{tabular}

Partindo destes achados, com o auxílio da NANDA-I e da CIPE, foram traçados os $\mathrm{DE}$ mais pertinentes ao quadro clínico dos pacientes com a COVID-19. Os DE mais prevalentes foram organizados e separados de acordo com a taxonomia utilizada na elaboração. Os diagnósticos da NANDA-I foram apresentados por domínio (Quadro 3).

Quadro 3 - Principais Diagnósticos de Enfermagem em indivíduos com a COVID-19. CratoCE, Brasil, 2020.

\begin{tabular}{|l|}
\multicolumn{1}{|c|}{ DIAGNÓSTICOS DE ENFERMAGEM DA NANDA-I } \\
\hline DOMÍNIO 1. Promoção da saúde \\
\hline $\begin{array}{l}\text { - Proteção ineficaz relacionado à incapacidade de proteção contra agente infeccioso } \\
\text { evidenciado por tosse, calafrios e fadiga }\end{array}$ \\
\hline DOMíNIO 2. Nutrição \\
\hline - Risco de desequilíbrio eletrolítico relacionado a presença de vômito e diarreia \\
\hline - Volume de líquidos excessivo relacionado a retenção hídrica evidenciado por edema em \\
membros inferiores \\
\hline DOMínIO 3. Eliminação e troca \\
\hline - Diarreia relacionada a processo infeccioso evidenciada por dor abdominal \\
\hline - Troca de gases prejudicada relacionada ao comprometimento pulmonar evidenciado por \\
dispneia e padrão respiratório anormal
\end{tabular}




\begin{tabular}{|l|}
\hline DOMÍNIO 4. Atividade/ repouso \\
\hline - Débito cardíaco diminuído relacionado a processo patológico evidenciado por palpitações \\
cardíacas e dispneia \\
\hline - Fadiga relacionada a processo patológico evidenciada por aumento dos sintomas físicos \\
\hline - Padrão respiratório ineficaz relacionado a dor e fadiga evidenciado por dispneia \\
\hline - Ventilação espontânea prejudicada relacionado a fadiga da musculatura respiratória \\
evidenciada por dispneia \\
\hline DOMÍNIO 10. Princípios de vida \\
\hline - Sofrimento espiritual relacionado a depressão, estressores e mudança ambiental \\
evidenciado por insônia \\
\hline DOMÍNIO 11. Segurança/ proteção \\
\hline - Hipertermia relacionada a processo infeccioso evidenciado por pele quente ao toque \\
\hline DOMínIO 12. Conforto \\
\hline - Dor aguda relacionada a agente biológico lesivo evidenciada por relato de comportamento \\
de dor/alterações nas atividades \\
\hline - Náusea relacionada a exposição a toxinas evidenciada por ânsia de vômito \\
\hline \multicolumn{1}{c|}{ DIAGNÓSTICOS DE ENFERMAGEM DA CIPE® } \\
\hline - Dor muscular \\
\hline - Dor musculoesquelética \\
\hline - Dor no peito \\
\hline - Presença de corrimento nasal \\
\hline - Presença de dispneia \\
\hline - Presença de edema em pernas \\
\hline - Presença de febre \\
\hline - Presença de hipertermia \\
\hline - Presença de tosse \\
\hline - Presença de vômito e diarreia \\
\hline
\end{tabular}

\section{DISCUSSÃO}

O Diagnóstico de Enfermagem constitui a segunda parte do Processo de Enfermagem (PE), consistindo no julgamento clínico das respostas humanas apresentadas pelo cliente, sendo uma parte essencial no traçado do plano de cuidados ao indivíduo em seu processo saúde-doença ${ }^{3,6}$.

Dentro deste contexto, encontra-se as taxonomias de enfermagem, cujo objetivo é padronizar a linguagem diagnóstica utilizada pelo profissional enfermeiro, sendo que entre elas se destaca a NANDA-I e a CIPE $\circledast^{36}$.
Para a construção dos enunciados diagnósticos da NANDA-I, é necessário que todo diagnóstico tenha um título com uma definição compreensível, além de que devem conter os indicadores diagnósticos, que podem ser as características definidoras, os fatores relacionados e os fatores de risco ${ }^{6}$.

Os diagnósticos de enfermagem da CIPE $®$ segue um Modelo de Sete Eixos - Cliente, Tempo, Ação, Julgamento, Localização, Foco e Meios, onde, para a elaboração do diagnóstico/resultado de enfermagem é obrigatório o uso de termos do Eixo Foco e do Eixo Julgamento, podendo ser inclusos termos 
de outros Eixos, não podendo ser incluído descritores do Eixo Ação, em contrapartida, para a construção das intervenções deve conter um termo do Eixo Ação, mas não pode haver descritor do Eixo Julgamento ${ }^{37}$.

O quadro clínico nos casos de infecção por COVID-19 é amplo, abrangendo desde casos assintomáticos até óbitos. Em suma, os principais sinais e sintomas identificados foram tosse, hipertermia, mialgia, calafrios, dificuldade respiratória, dor no peito, dispneia e expectoração produtiva, além de sintomas gastrointestinais e emocionais também estarem comumente presentes $^{2,10,16,31-33}$.

Com isso, os diagnósticos de enfermagem construídos servem como guia da prática clínica da assistência dos enfermeiros no contexto dos pacientes acometidos com a infecção por SARSCov-2.

O DE Proteção Ineficaz diz respeito à incapacidade do organismo se defender de perigos internos ou externos. Dentro do contexto dos pacientes com COVID-19, está relacionado aos sinais e sintomas de tosse, linfocitopenia, dispenia e fadiga, como apresentado em alguns casos de pacientes contaminados $6,10,13,19$ -

$22,25,27,28,31,34$.
A presença de sintomas gastrointestinais, tais como diarreia e vômitos, cujas consequências podem incluir perda de eletrólitos essenciais para a manutenção da homeostase corporal, em alguns casos de infecção pelo novo coronavírus, caracterizam o diagnóstico de Risco de Desequilíbrio Eletrolítico, Diarreia e Presença de Vômito e Diarreia. O DE Volume de Líquidos Excessivo e Presença de Edema em Pernas também se faz presente devido à presença de retenção hídrica percebida em membros inferiores de pacientes renais acometidos com a atual afecção. Dentro deste contexto, faz-se também presente o DE Náusea, cuja principal característica é o autorrelato por parte dos pacientes ao sentir o sintoma ${ }^{6,7,13,38,39}$.

O DE Troca de Gases Prejudicada é bastante comum em pacientes acometidos por pneumonias, algo característico da infecção pelo SARSCov-2, resultando, na maioria das vezes em dificuldade respiratória (dispneia), resultando, por conseguinte nos diagnósticos de Padrão Respiratório Ineficaz e Presença de Dispneia inseridos dentro do quadro dos pacientes ${ }^{6,7,40}$.

Quando a dispneia se torna mais intensa, necessitando de intervenção como a oxigenoterapia ou a Ventilação 
Mecânica Invasiva (MIC), é necessário aplicar o diagnóstico de Ventilação Espontânea Prejudicada, cuja definição é a incapacidade de iniciar e/ou manter um padrão respiratório que seja capaz de sustentar a vida, sendo que na prática clínica este diagnóstico é comumente mais aplicado em clientes em uso de Ventilação Mecânica Invasiva ${ }^{6,41}$.

Referente aos exames clínicos realizados em pacientes com infecção pelo SARS-Cov-2, a Tomografia Computadorizada (TC) foi muito utilizada, tendo opacidade em vidro fosco como principal achado, o que evidencia severo comprometimento pulmonar. Este achado pode justificar a dificuldade respiratória e alguns casos de pneumonia relacionada ao uso da $\mathrm{VM}^{10,16,18,24,26,29,30,35}$.

Além disso, a dispneia pode ser resultado de alterações cardiovasculares, como descrito no DE Débito Cardíaco Diminuído, como o aumento do volume intravascular, podendo causar, inclusive, edema pulmonar, o que também provocaria o quadro de tosse. Este diagnóstico, bem como o DE Dor no Peito, estão presentes no contexto dos pacientes que apresentam o sintoma de palpitações no peito, um achado encontrado em alguns pacientes infectados por SARS-Cov-2, além de que a também presença edema é um sinal indicativo deste diagnóstico de enfermagem $6,7,12,13,17,22,23,26,42,43$.

O diagnóstico de Fadiga pode ser definido como um evento multicausal e subjetivo onde estão integrados aspectos tanto físicos, quanto emocionais e cognitivos, sendo que pode ser manifestada na forma de falta de energia corporal e sensação de cansaço relatado pelo cliente, possuindo também como fatores relacionados o estado físico fragilizado e os estágios da doença, portanto, este diagnóstico enquadra-se no contexto do COVID-19 devido a condição debilitante na qual os pacientes se encontram ${ }^{44,45}$.

A definição para o DE Sofrimento Espiritual é a de incapacidade de atribuir ou mesmo experimentar objetivo e/ou significado através de conexão consigo mesmo, com outras pessoas, com a arte, música e literatura, com a natureza ou com um ser superior e está relacionado ao fato de que os pacientes acometidos pela infecção por SARS-Cov-2 necessitarem de isolamento para impedir transmissão do vírus, isto é mudança ambiental, o que gera estressores, o que pode resultar em depressão e insônia, como mostrado em alguns estudos ${ }^{11,46}$. 
O conceito para hipertermia é dado da seguinte forma: temperatura corporal superior aos limites considerados normais durante o período diurno sendo o resultado de falhas no sistema termorregulador. Alguns fatores clínicos relacionados a este DE são pele quente ao toque e pele avermelhada. Este diagnóstico surge em pacientes com COVID-19 por se tratar de um sintoma frequentemente presente nesta afecção, assim o são os DE Presença de Febre e Presença de Hipertermia $6,11,14,16,17,20-$ $23,27,32,47$.

O DE dor aguda pode ser interpretado como o autorrelato do cliente a respeito de uma experiência sensorial indesejável associada a lesão tecidual real ou potencial, de início repentino ou gradual, na qual pode ser solicitado a quantificação de sua intensidade por meio de utilização de escalas padronizadas, podendo ser leve ou intensa, cujo início seja menor do que três meses. Os fatores relacionados podem ser por agentes biológicos (por exemplo, agente infeccioso), agentes químicos (a exemplo da capsaicina) e/ou por agentes físicos (como trauma). No contexto dos pacientes acometidos por infecção por SARS-Cov-2, dá-se pelo fato de muitos destes apresentarem mialgia como um dos achados clínicos, sendo que os diagnósticos de Dor Muscular e Dor Musculoesquelética também se enquadram neste contexto6,12,14,18,21,23,48. Ademais, a tosse e corrimento nasal são manifestações clínicas relativamente comuns entre as pessoas acometidas pela COVID-19, portanto os DE Presença de Tosse e Presença de Corrimento Nasal fazem-se presentes dentro deste quadro $7,10,14,19,21,22,25,27,28,33,34$.

Em suma, o momento é de muitas incertezas, em curto e longo prazo. Dessa forma, destaca-se a importância de ser prestada uma assistência individualizada; principalmente por parte da equipe de enfermagem, mais frequentemente envolvida no processo do cuidar e seguimento terapêutico dos pacientes. Nesse sentido, é fundamental a aplicação do processo de enfermagem, com destaque para a identificação de $\mathrm{DE}$ entre esses indivíduos, a fim de alcançar todas as necessidades humanas apresentadas, favorecendo uma prática sistematizada e holística.

Como fatores limitantes para realização desse estudo, a ausência de pesquisas publicadas sobre DE para vítimas do novo coronavírus. Entretanto, conseguiu-se alcançar o principal objetivo desta investigação. 


\section{CONSIDERAÇÕES FINAIS}

Os principais sinais e sintomas identificados nos casos de COVID-19 foram tosse, hipertermia, e expectoração produtiva, além de calafrios e mialgia. Em alguns indivíduos, notou-se presença de dificuldade respiratória, e alguns casos evoluíram para óbito.

Foram identificados 25 diagnósticos de enfermagem traçados a partir da NANDA-I e da CIPE, de acordo com o quadro clínico mais presente. Os diagnósticos prevalentes foram proteção ineficaz relacionado à incapacidade de proteção contra agente infeccioso evidenciado por tosse, calafrios e fadiga; dor muscular; dor musculoesquelética; padrão respiratório ineficaz relacionado à dor e fadiga evidenciado por dispneia, e sofrimento espiritual relacionado a depressão, estressores e mudança ambiental evidenciado por insônia.

Entre esses, sobressaiu o diagnóstico sofrimento espiritual; importante achado que pode contribuir para o alcance de um olhar profissional que ultrapasse os cuidados somente às dimensões físicas.

0 estudo traz importante contribuição para a prática clínica dos enfermeiros, bem como de toda a equipe, uma vez que a implementação da Sistematização da Assistência de Enfermagem e do Processo de Enfermagem conferem impactos positivos para o cuidado ao paciente.

\section{REFERÊNCIAS}

1. Ministério da Saúde (BR). O que é coronavírus?. [Internet]. Brasília: Ministério da Saúde; 2020 [acesso em 05 abr 2020]. Disponível em: https: / / www.saude.gov.br/o ministro/746-saude-de-a-a-z/46490novo-coronavirus-o-que-e-causassintomas-tratamento-e-prevencao.

2. Mendonça FD, Rocha SS, Pinheiro DLP, Oliveira SV. Região Norte do Brasil e a pandemia de COVID-19: análise socioeconômica e epidemiológica. J Health NPEPS. 2020; 5(1):20-37.

3. Ministério da Saúde (BR). Boletim CEO Covid 19 n. 13. [Internet]. Brasília: Ministério da Saúde; 2020 [acesso em 24 abr 2020]. Disponível em:https://coronavirus.saude.gov.br /index.php/boletins-epidemiologicos.

4. Barros ALBL, Lopes JL. A legislação e a sistematização da assistência de enfermagem. Enferm Foco. 2010; 1(2):63-65. 
5. Horta WA. Enfermagem: teoria, conceitos, princípios e processo. Rev Esc Enferm USP. 1974; 5(1):7-15.

6. Herdman TH, Kamitsuru S. Diagnósticos de enfermagem da NANDA-I: definições e classificação 2018-2020 [Internet]. Porto Alegre: NANDA International; [acesso em 26 abr 2020]. Disponível em: http://nascecme.com.br/2014/wpco ntent/uploads/2018/08/NANDA-I2018_2020.pdf.

7. Conselho Internacional de Enfermagem. Classificação Internacional para a Prática de Enfermagem: $\quad$ CIPE $®$. Versão 2019/2020. 2019; 1-182.

8. Mendes KDS, Silveira RCCP, Galvão CM. Uso de gerenciador de referências bibliográficas na seleção dos estudos primários em revisão integrativa. Texto \& Contexto Enferm. 2019; 28(1):1-13.

9. Moher D, Liberati A, Tetzlaff J, Altman DJ. Preferred Reporting Items for Systematic Reviews and MetaAnalyses: The PRISMA Statement. PLoS Med. 2009; 6(7):1-7.

10. Zhou F, Yu T, Du R, Fan G, Liu Y, Liu $Z$, et al. Clinical course and risk factors for mortality of adult inpatients with COVID-19 in Wuhan,
China: a retrospective cohort study. The Lancet. 2020; 395:1054-1062.

11. Lim J, Jeon S, Shin HY, Kim MJ, Seong YM, Lee WJ, et al. Case of the Index Patient Who Caused Tertiary Transmission of Coronavirus Disease 2019 in Korea: the Application of Lopinavir/Ritonavir for the Treatment of COVID-19 Pneumonia Monitored by Quantitative RT-PCR. J Korean Med Sci. 2020; 35(6):1-6.

12. Chen Y, Guo J, Wang C, Luo F, Yu X, Zhang $W$, et al. Clinical characteristics and intrauterine vertical transmission potential of COVID-19 infection in nine pregnant women: a retrospective review of medical records. The Lancet. 2020; 12:1-7.13.

13. Fu D, Yang Bo, Xu J, Mao Z, Zhou C, Xue C. COVID-19 Infection in a Patient with End-Stage Kidney Disease. Nephron. 2020; 144(5):245247.

14. Sun R, Liu H, Wang X. Mediastinal Emphysema, Giant Bulla, and Pneumothorax Developed during the Course of COVID-19 Pneumonia. Korean J Radiol. 2020; 21(5): 541544.

15. Park JY, Ham MS, Park KU, Kim JY, Choi EH. First Pediatric Case of 
Coronavirus Disease 2019 in Korea. J Korean Med Sci. 2020; 35(11):1-7.

16. Ruan Q, Yang K, Wang W, Jiang L, Song J. Clinical predictors of mortality due to COVID-19 based on an analysis of data of 150 patients from Wuhan, China. Intensive Care Med. 2020; 46(5):846-848.

17. Cuong LV, Giang HTN, Linh LK, Shah J, Sy LV, Hung TH, et al. The first Vietnamese case of COVID-19 acquired from China. Lancet Infect Dis. 2020; 20(4):408-409.

18. Sellevoll HB, Saeed U, Young VS, Sandbaek G, Gundersen K, Mala T. Acute abdomen as an early symptom. Tidsskr Nor Laegeforen. 2020; 140(7):1-6.

19. Ihle-Hansen $H$, Berge $T$, Tveita $A$, Rønning EJ, Ernø PE, Andersen EL, et al. COVID-19: Symptoms, course of illness and use of clinical scoring systems for the first 42 patients admitted to a Norwegian local hospital. Tidsskr Nor Laegeforen. 2020; 140(7):1-10. 20.

20. Ottestad W, Seim M, Mæhlen JO. COVID-19 with silent hypoxemia. Tidsskr Nor Laegeforen. 2020; 140(7):1-11.

21. Wang D, Yin Y, Hu C, Liu X, Zhang X, Shuliang Zhou, et al. Clinical Course and Outcome of 107 Patients
Infected With the Novel Coronavirus, SARS-CoV-2, Discharged From Two Hospitals in Wuhan, China. Crit Care. 2020; 24(1):1-9.

22. Zhao $X Y$, $X u X X$, Yin $H S$, Hu QM, Xiong $\mathrm{T}$, Tang $\mathrm{YY}$, et al. Clinical characteristics of patients with 2019 coronavirus disease in a non-Wuhan área of Hubei Province, China: a retrospective study. BMC Infectious Diseases. 2020; 20(311):1-8.

23. Xin T, Juan H, Fen Z, Yan Z, Jieqiong L, Xiangyun W. Analysis of the clinical characteristics of 13 cases of new coronavirus infection in children in Changsha. Zhongguo Dang Dai Er ke Za Zhi. 2020; 22(4):294-298.

24. Righi G, Popolo GD. COVID-19 tsunami: the first case of a spinal cord injury patient in Italy. Spinal Cord Ser Cases. 2020; 6(1):1-5.

25. Breslin N, Batista C, GyamfiBannerman C, Miller R, Martinez R, Bernstein K., et al. Coronavirus disease 2019 infection among asymptomatic and symptomatic pregnant women: two weeks of confirmed presentations to an affiliated pair of New York City hospitals. Am J Obstet Gynecol MFM. 2020; 2(2).

26. Azwar MK, Kirana F, Kurniawan A, Handayani S, Setiati S. 
Gastrointestinal Presentation in COVID-19 in Indonesia: A Case Report. Acta Med Indones - Indones J Intern Med. 2020; 52(1):63-67.

27. Zheng F, Tang W, Li H, Huang YX, Xie YL, Zhou ZG. Clinical characteristics of 161 cases of corona virus disease 2019 (COVID-19) in Changsha. Eur Rev Med Pharmacol Sci. 2020; 24(6):3404-3410.

28. Su L, Ma X, Yu H, Zhang Z, Bian P, Han $Y$, et al. The different clinical characteristics of corona virus disease cases between children and their families in China - the character of children with COVID-19. Emerg Microbes Infect. 2020; 9(1):707-713.

29. Shi F, Yu Q, Huang W, Tan C. 2019 Novel Coronavirus (COVID-19) Pneumonia with Hemoptysis as the Initial Symptom: CT and Clinical Features. Korean J Radiol. 2020; 21(5):537-540.

30. Xia W, Shao J, Guo Y, Peng X, Li Z, $\mathrm{Hu}$ D. Clinical and CT features in pediatric patients with COVID-19 infection: Different points from adults. Pediatric Pulmonology. 2020; 55:1169-1174.

31. Li K, Wu J, Wu F, Guo D, Chen L, Fang $Z$, et al. The Clinical and Chest CT Features Associated With Severe and Critical COVID-19 Pneumonia. Invest Radiol. 2020; 55(6):1-5.

32. Yang W, Cao Q, Qin L, Wang X, Cheng Z, Pan A, et al. Clinical characteristics and imaging manifestations of the 2019 novel coronavirus disease (COVID-19):A multi-center study in Wenzhou city, Zhejiang, China. J Infect. 2020; 80(4):388-393.

33. Xu XW, Wu XX, Jiang XG, Xu KJ, Ying $\mathrm{LJ}, \mathrm{Ma} \mathrm{CL}$, et al. Clinical findings in a group of patients infected with the 2019 novel coronavirus (SARS-Cov-2) outside of Wuhan, China: retrospective case series. BMJ. Feb 2020.

34. Liu K, Fang YY, Deng Y, Liu W, Wang MF, Ma JP, et al. Clinical characteristics of novel coronavirus cases in tertiary hospitals in Hubei Province. Chin Med J (Engl). 2020; 133(9):1025-1031.

35. Wang Z, Chen X, Lu Y, Chen F, Zhang W. Clinical characteristics and therapeutic procedure for four cases with 2019 novel coronavirus pneumonia receiving combined Chinese and Western medicine treatment. Biosci Trends. 2020; 14(1):64-68.

36. Ferreira AM, Rocha EN, Lopes CT, Bachion MM, Lopes JL, Barros ALBL. 
Diagnósticos de enfermagem em terapia intensiva: mapeamento cruzado e Taxonomia da NANDA-I. Rev Bras Enferm. 2016; 69(2):307-15. 37. Félix NDC. Diagnósticos/resultados de enfermagem da CIPE $®$ para pessoas com síndrome metabólica. [Dissertação] Crato (BR): Universidade Regional do Cariri; 2016.

38. Clares JWB, Freitas MC. Diagnósticos de enfermagem do domínio Nutrição identificados em idosos da comunidade. Rev Eletrônica Enferm. 2013; 15(4):940-7.

39. Santos AMS, Campelo SMA, Santos WN, Silva RAR. Nursing diagnoses in patients with nephropathies. Rev Enferm UFPI. 2017; 6(4):65-9.

40. Araújo AAO, Pascoal LM, Gontijo PVC, Lopes MVO, Santos Neto M, Nunes SFL. Acurácia e prevalência das características definidoras do diagnóstico Troca de gases prejudicada em crianças. Rev Rene. 2019; 20:1-8.

41. Seganfredo DH, Beltrão BA, Silva VM, Lopes MVO, Castro SMJ, Almeida MA. Análise do padrão respiratório ineficaz e ventilação espontânea prejudicada de adultos com oxigenoterapia. Rev Latinoam Enferm. 2017; 25:1-9.
42. Sousa AMM, Lima ABS, Pascoal LM, Rouberte ESC, Rolim ILTP. Débito cardíaco diminuído: mapeamento cruzado das intervenções de enfermagem e sua contribuição na prática clínica. Enferm Glob. 2019; (56):338-351.

43. Ribeiro IA, Lima LR, Volpe CRG, Funghetto SS, Rehem TCMSB, Stival MM. Síndrome do idoso frágil em idosos com doenças crônicas na Atenção Primária. Rev Esc Enferm USP. 2019; 53:1-9.

44. Macedo BM. Acuracia dos indicadores clínicos do diagnóstico de Enfermagem fadiga em pacientes com insuficiência cardíaca. [Dissertação] Natal(BR): Universidade Federal do Rio Grande do Norte; 2019.

45. Silva RAR, Bezerra MX, Souza Neto VL, Mororo DDS, Andrade ICF. Crianças com doenças renais: associação entre diagnósticos de enfermagem e seus componentes. Acta Paul Enferm. 2017; 30(1):73-9.

46. Lenne A, Fernandes RAQ, Puggina AC. A espiritualidade de enfermeiros assistenciais interfere no registro do diagnóstico sofrimento espiritual? Esc Anna Nery. 2018; 22(1):1-10.

47. Braga FC, Santos ARC, Castro NB, Nunes MM, Lopes MVO, Silva VM. 
Acurácia dos indicadores clínicos dos diagnósticos de Enfermagem hipertermia e hipotermia em recémnascidos. Rev Rene. 2014; 15(5):78995.
48. Correia MDL, Duran ECM. Definição conceitual e operacional dos componentes do diagnóstico de enfermagem Dor Aguda. Rev Latinoam Enferm. 2017; 25:1-10.

\section{ANEXO}

Quadro 1 - Características dos estudos selecionados. Crato-CE, Brasil, 2020.

\begin{tabular}{|c|c|c|c|}
\hline TÍTULO & $\begin{array}{l}\text { AUTORES/ ANO DE } \\
\text { PUBLICAÇÃO }\end{array}$ & OBJETIVO & AMOSTRA \\
\hline $\begin{array}{l}\text { Clinical course and } \\
\text { risk factors for } \\
\text { mortality of adult } \\
\text { inpatients rith } \\
\text { COVID-19 in Wuhan, } \\
\text { China: r a } \\
\text { retrospective cohort } \\
\text { study. }\end{array}$ & $\begin{array}{l}\text { Zhou F, Yu T, Du R, Fan } \\
\text { G, Liu Y, Liu Z, et al. } \\
(2020)\end{array}$ & $\begin{array}{lr}\text { Explorar os } & \text { fatores } \\
\text { de riscos } & \text { para } \\
\text { mortalidade e } & \text { o } \\
\text { curso clínico } & \text { do } \\
\text { COVID-19 } & \text { em } \\
\text { pacientes } & \\
\text { internados. } & \\
\end{array}$ & $\begin{array}{l}191 \text { pacientes com } \\
\text { idade } \geq 18 \text { anos com } \\
\text { diagnóstico de COVID- } \\
19 .\end{array}$ \\
\hline $\begin{array}{l}\text { Case of the Index } \\
\text { Patient Who Caused } \\
\text { Tertiary } \\
\text { Transmission of } \\
\text { Coronavirus Disease } \\
2019 \text { in Korea: the } \\
\text { Application of } \\
\text { Lopinavir/Ritonavir } \\
\text { for the Treatment of } \\
\text { COVID-19 Pneumonia } \\
\text { Monitored by } \\
\text { Quantitative RT- } \\
\text { PCR. }\end{array}$ & $\begin{array}{l}\text { Lim J, Jeon S, Shin HY, } \\
\text { Kim MJ, Seong YM, Lee } \\
\text { WJ, et al. (2020) }\end{array}$ & 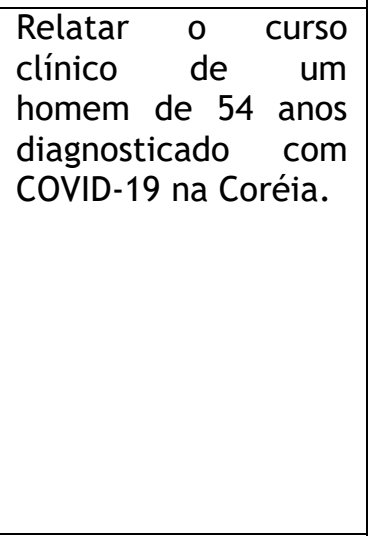 & $\begin{array}{l}\text { Paciente índice com } \\
\text { COVID-19 que causou } \\
\text { infecção terciária, } \\
\text { sendo logo em seguida } \\
\text { isolado. }\end{array}$ \\
\hline $\begin{array}{l}\text { Clinical } \\
\text { characteristics and } \\
\text { intrauterine vertical } \\
\text { transmission } \\
\text { potential of COVID- } \\
19 \text { infection in nine } \\
\text { pregnant women: a } \\
\text { retrospective review } \\
\text { of medical records. }\end{array}$ & $\begin{array}{l}\text { Chen Y, Guo J, Wang C, } \\
\text { Luo F, Yu X, Zhang W, } \\
\text { et al. (2020) }\end{array}$ & $\begin{array}{lr}\text { Avaliar } & \text { as } \\
\text { características } & \\
\text { clínicas do COVID-19 } \\
\text { na gravidez } & \text { e } \\
\text { potencial } & \text { de } \\
\text { transmissão } & \text { vertical } \\
\text { intra-uterino } & \text { da } \\
\text { infecção por } & \text { COVID- } \\
19 . & \end{array}$ & $\begin{array}{l}\text { Nove mulheres grávidas } \\
\text { que testaram positivo } \\
\text { para o novo coronavírus } \\
\text { internadas com grave } \\
\text { síndrome respiratória } \\
\text { aguda. }\end{array}$ \\
\hline $\begin{array}{l}\text { COVID-19 Infection } \\
\text { in a Patient with } \\
\text { End-Stage Kidney } \\
\text { Disease. }\end{array}$ & $\begin{array}{l}\text { Fu D, Yang Bo, Xu J, } \\
\text { Mao Z, Zhou C, Xue C. } \\
(2020)\end{array}$ & $\begin{array}{l}\text { Relatar o caso de um } \\
\text { homem de } 75 \text { anos } \\
\text { que tem doença } \\
\text { renal } \\
\text { infectado crônica } \\
\text { novo com o o }\end{array}$ & $\begin{array}{l}\text { Paciente com } 75 \text { anos } \\
\text { de idade, portador de } \\
\text { doença renal crônica } \\
\text { em estágio terminal. }\end{array}$ \\
\hline
\end{tabular}




\begin{tabular}{|c|c|c|c|}
\hline $\begin{array}{l}\text { Mediastinal } \\
\text { Emphysema, Giant } \\
\text { Bulla, and } \\
\text { Pneumothorax } \\
\text { Developed during } \\
\text { the Course of COVID- } \\
19 \text { Pneumonia. }\end{array}$ & $\begin{array}{l}\text { Sun R, Liu H, Wang X. } \\
(2020)\end{array}$ & 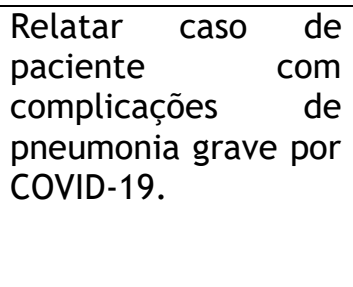 & $\begin{array}{l}\text { Homem de } 38 \text { anos com } \\
\text { COVID-19, cujo curso } \\
\text { clínico teve enfisema } \\
\text { mediastinal e formação } \\
\text { de bolha no pulmão e } \\
\text { pneumotórax. }\end{array}$ \\
\hline $\begin{array}{lr}\text { First } & \text { Pediatric } \\
\text { of } & \text { Coronave } \\
\text { Disease } 2019 \text { in } \\
\text { Korea. }\end{array}$ & $\begin{array}{l}\text { Park JY, Ham MS, Park } \\
\text { KU, Kim JY, Choi EH. } \\
(2020)\end{array}$ & $\begin{array}{l}\text { Relatar o primeiro } \\
\text { caso pediátrico de } \\
\text { COVID-19 na Coréia. }\end{array}$ & $\begin{array}{l}\text { Menina de } 10 \text { anos } \\
\text { infectada por COVID-19. }\end{array}$ \\
\hline $\begin{array}{l}\text { Clinical predictors of } \\
\text { mortality due to } \\
\text { COVID-19 based on } \\
\text { an analysis of data } \\
\text { of } 150 \text { patients from } \\
\text { Wuhan, China. }\end{array}$ & $\begin{array}{l}\text { Ruan Q, Yang K, Wang } \\
\text { W, Jiang L, Song J. } \\
(2020)\end{array}$ & $\begin{array}{l}\text { Relatar dados } \\
\text { demográficos, } \\
\text { características } \\
\text { clínicas e resultados } \\
\text { laboratoriais de } \\
\text { pacientes internados } \\
\text { com diagnóstico de } \\
\text { COVID-19. }\end{array}$ & $\begin{array}{l}150 \text { pacientes } \\
\text { diagnosticados com } \\
\text { coronavírus em Wuhan, } \\
\text { China. }\end{array}$ \\
\hline $\begin{array}{lr}\text { The first Vietnamese } \\
\text { case of } & \text { COVID-19 } \\
\text { acquired } & \text { from } \\
\text { China. } & \\
\end{array}$ & $\begin{array}{l}\text { Cuong LV, Giang HTN, } \\
\text { Linh LK, Shah J, Sy LV, } \\
\text { Hung TH, et al. (2020) }\end{array}$ & $\begin{array}{l}\text { Descrever o primeiro } \\
\text { caso de COVID-19 do } \\
\text { Vietnã adquirido na } \\
\text { China. }\end{array}$ & $\begin{array}{l}\text { Uma vietnamita de } 25 \\
\text { anos que esteve em } \\
\text { Wuhan na China. }\end{array}$ \\
\hline $\begin{array}{l}\text { Acute abdomen as } \\
\text { an early symptom } \\
\text { of COVID-19 }\end{array}$ & $\begin{array}{l}\text { Sellevoll HB, Saeed U, } \\
\text { Young VS, Sandbaek G, } \\
\text { Gundersen K, Mala T. } \\
(2020)\end{array}$ & $\begin{array}{lr}\text { Relatar } & \text { as } \\
\text { características } & \\
\text { clínicas de uma } \\
\text { mulher } \\
\text { diagnosticada } \\
\text { COVID-19. }\end{array}$ & $\begin{array}{lcc}\text { Paciente com } & \text { dor } \\
\text { abdominal aguda. } & \end{array}$ \\
\hline $\begin{array}{l}\text { COVID-19: } \\
\text { Symptoms, course of } \\
\text { illness and use of } \\
\text { clinical scoring } \\
\text { systems for the first } \\
42 \text { patients admitted } \\
\text { to a Norwegian local } \\
\text { hospital. }\end{array}$ & $\begin{array}{l}\text { Ihle-Hansen H, Berge T, } \\
\text { Tveita A, Rønning EJ, } \\
\text { Erno PE, Andersen EL, } \\
\text { et al. (2020) }\end{array}$ & $\begin{array}{lr}\text { Apresentar } & \text { os } \\
\text { sintomas, } & \text { achados } \\
\text { clínicos e evolução } \\
\text { do COVID-19 em } \\
\text { pacientes } \\
\text { internados. }\end{array}$ & $\begin{array}{l}42 \text { pacientes internados } \\
\text { em um hospital } \\
\text { norueguês. }\end{array}$ \\
\hline $\begin{array}{l}\text { COVID-19 with silent } \\
\text { hypoxemia. }\end{array}$ & $\begin{array}{l}\text { Ottestad W, Seim M, } \\
\text { Mæhlen JO. (2020) }\end{array}$ & $\begin{array}{l}\text { Descrever o caso de } \\
\text { um paciente com } \\
\text { COVID-19 que } \\
\text { evoluiu rapidamente } \\
\text { para insuficiência } \\
\text { respiratória. }\end{array}$ & $\begin{array}{l}\text { Homem de } 60 \text { anos com } \\
\text { COVID-19 que evoluiu } \\
\text { para quadro de } \\
\text { insuficiência } \\
\text { respiratória. }\end{array}$ \\
\hline $\begin{array}{l}\text { Clinical course and } \\
\text { outcome of } 107 \\
\text { patients infected } \\
\text { with the novel } \\
\text { coronavirus, SARS- } \\
\text { CoV-2, discharged } \\
\text { from two hospitals in } \\
\text { Wuhan, China. }\end{array}$ & $\begin{array}{l}\text { Wang D, Yin Y, Hu C, } \\
\text { Liu X, Zhang X, Shuliang } \\
\text { Zhou, et al. (2020) }\end{array}$ & $\begin{array}{l}\text { Descrever o curso } \\
\text { clínico e prognóstico } \\
\text { de pacientes com } \\
\text { COVID-19. }\end{array}$ & $\begin{array}{lr}107 & \text { pacientes } \\
\text { diagnosticados } & \text { com } \\
\text { COVID-19. } & \end{array}$ \\
\hline $\begin{array}{l}\text { Clinical } \\
\text { characteristics of } \\
\text { patients with } 2019 \\
\text { coronavirus disease } \\
\text { in a non-Wuhan area } \\
\text { of Hubei Province, } \\
\text { China: a } \\
\text { retrospective study. }\end{array}$ & $\begin{array}{l}\text { Zhao XY, Xu XX, Yin HS, } \\
\text { Hu QM, Xiong T, Tang } \\
\text { YY, et al. (2020) }\end{array}$ & $\begin{array}{l}\text { Descrever as } \\
\text { características } \\
\text { clínicas de pacientes } \\
\text { com COVID-19 em } \\
\text { áreas fora de } \\
\text { Wuhan, província de } \\
\text { Hubei, na China. }\end{array}$ & $\begin{array}{l}91 \text { pacientes } \\
\text { diagnosticados } \quad \text { com } \\
\text { COVID-19. }\end{array}$ \\
\hline
\end{tabular}




\begin{tabular}{|c|c|c|c|}
\hline $\begin{array}{l}\text { Analysis of the } \\
\text { clinical } \\
\text { characteristics of } 13 \\
\text { cases of new } \\
\text { coronavirus infection } \\
\text { in children in } \\
\text { Changsha. }\end{array}$ & $\begin{array}{l}\text { Xin T, Juan H, Fen Z, } \\
\text { Yan Z, Jieqiong L, } \\
\text { Xiangyun W. (2020) }\end{array}$ & $\begin{array}{lr}\begin{array}{l}\text { Analisar } \\
\text { características }\end{array} & \text { as } \\
\text { clínicas da } & \text { nova } \\
\text { infecção } & \text { por } \\
\text { coronavírus infantil. }\end{array}$ & $\begin{array}{l}13 \text { crianças infectadas } \\
\text { com o vírus SARS-CoV-2 } \\
\text { em Changsha. }\end{array}$ \\
\hline $\begin{array}{l}\text { COVID-19 tsunami: } \\
\text { the first case of a } \\
\text { spinal cord injury } \\
\text { patient in Italy. }\end{array}$ & $\begin{array}{l}\text { Righi G, Popolo GD. } \\
(2020)\end{array}$ & $\begin{array}{l}\text { Relatar o primeiro } \\
\text { caso de uma pessoa } \\
\text { tetraplégica com } \\
\text { COVID-19 na Itália. }\end{array}$ & $\begin{array}{l}\text { Homem de } 56 \text { anos com } \\
\text { tetraplegia } \\
\text { diagnosticado } \\
\text { COVID-19. }\end{array}$ \\
\hline $\begin{array}{l}\text { Coronavirus disease } \\
2019 \text { infection } \\
\text { among asymptomatic } \\
\text { and symptomatic } \\
\text { pregnant women: } \\
\text { two weeks of } \\
\text { confirmed } \\
\text { presentations to an } \\
\text { affiliated pair of } \\
\text { New York City } \\
\text { hospitals. }\end{array}$ & $\begin{array}{l}\text { Breslin N, Batista } \text { C, } \\
\text { Gyamfi-Bannerman C, } \\
\text { Miller R, Martinez R, } \\
\text { Bernstein K, et al. } \\
(2020)\end{array}$ & $\begin{array}{l}\text { Descrever uma série } \\
\text { de casos de } \\
\text { pacientes grávidas } \\
\text { confirmadas para } \\
\text { com COVID-19. }\end{array}$ & $\begin{array}{l}43 \text { gestantes que } \\
\text { testaram positivo para o } \\
\text { vírus do COVID- } 19 .\end{array}$ \\
\hline $\begin{array}{l}\text { Gastrointestinal } \\
\text { Presentation } \\
\text { COVID-19 in } \\
\text { Indonesia: A } \\
\text { Report. }\end{array}$ & $\begin{array}{l}\text { Azwar MK, Kirana F, } \\
\text { Kurniawan A, Handayani } \\
\text { S, Setiati S. (2020) }\end{array}$ & $\begin{array}{l}\text { Relatar o caso de um } \\
\text { paciente na } \\
\text { Indonésia com dor } \\
\text { no peito e sintomas } \\
\text { gastrointestinais } \\
\text { quando confirmou } \\
\text { ter infecção por } \\
\text { SARS-Cov-2. }\end{array}$ & $\begin{array}{l}\text { Homem com diagnóstico } \\
\text { de COVID-19 } \\
\text { apresentando dor no } \\
\text { peito e sintomas } \\
\text { gastrointestinais. }\end{array}$ \\
\hline $\begin{array}{lr}\text { Clinical } \\
\text { Characteristics of } \\
161 \text { cases of corona } \\
\text { virus disease } 2019 \\
\text { (COVID-19) } \\
\text { Changsha. }\end{array}$ & $\begin{array}{l}\text { Zheng F, Tang W, Li H, } \\
\text { Huang YX, Xie YL, Zhou } \\
\text { ZG. (2020) }\end{array}$ & $\begin{array}{l}\text { Investigar } \\
\text { características as } \\
\text { clínicas do } \\
\text { coronavírus doença } \\
\text { 2019 (COVID-19). }\end{array}$ & $\begin{array}{l}161 \text { casos de COVID-19 } \\
\text { admitidos em um } \\
\text { Hospital de Changsha. }\end{array}$ \\
\hline $\begin{array}{l}\text { The different clinical } \\
\text { characteristics of } \\
\text { corona virus disease } \\
\text { cases between } \\
\text { children and their } \\
\text { families in China - } \\
\text { the character of } \\
\text { children with COVID- } \\
19 .\end{array}$ & $\begin{array}{l}\text { Su L, Ma X, Yu H, Zhang } \\
\text { Z, Bian P, Han Y, et al. } \\
(2020)\end{array}$ & $\begin{array}{lr}\text { Analisar } & \text { as } \\
\text { diferentes } & \\
\text { características } & \\
\text { clínicas } & \text { entre } \\
\text { crianças e suas } \\
\text { famílias infectadas } \\
\text { com graves } & \\
\text { síndrome } & \\
\text { respiratória aguda } \\
\text { por COVID-19. }\end{array}$ & $\begin{array}{l}\text { Nove crianças e } 14 \\
\text { pacientes adultos. }\end{array}$ \\
\hline $\begin{array}{l}2019 \text { Novel } \\
\text { Coronavirus (COVID- } \\
\text { 19) Pneumonia with } \\
\text { Hemoptysis as the } \\
\text { Initial Symptom: CT } \\
\text { and Clinical } \\
\text { Features. }\end{array}$ & $\begin{array}{l}\text { Shi F, Yu Q, Huang W, } \\
\text { Tan C. (2020) }\end{array}$ & $\begin{array}{l}\text { Relatar o caso de um } \\
\text { paciente com } \\
\text { sintomas atípicos do } \\
\text { COVID-19. }\end{array}$ & $\begin{array}{l}\text { Homem de } 57 \text { anos } \\
\text { apresentando quadro de } \\
\text { hemoptise. }\end{array}$ \\
\hline $\begin{array}{lr}\text { Clinical and } & \text { CT } \\
\text { features in pediatric } \\
\text { patients r with } \\
\text { COVID-19 } \\
\text { infection: Different } \\
\text { points from adults. }\end{array}$ & $\begin{array}{l}\text { Xia W, Shao J, Guo Y, } \\
\text { Peng X, Li Z, Hu D. } \\
(2020)\end{array}$ & $\begin{array}{l}\text { Discutir as diferentes } \\
\text { características } \\
\text { clínicas, } \\
\text { laboratoriais e } \\
\text { tomografia } \\
\text { computadorizada }\end{array}$ & $\begin{array}{l}20 \text { pacientes pediátricos } \\
\text { com infecção por COVID } \\
-19 \text { confirmada. }\end{array}$ \\
\hline
\end{tabular}




\begin{tabular}{|c|c|c|c|}
\hline & & $\begin{array}{lr}\text { (TC) em pacientes } \\
\text { pediátricos } \\
\text { adultos de com novo } \\
\text { coronavírus de } 2019 .\end{array}$ & \\
\hline $\begin{array}{lr}\text { The Clinical and } \\
\text { Chest CT } & \text { Features } \\
\text { Associated } & \text { With } \\
\text { Severe and } & \text { Critical } \\
\text { COVID-19 } & \\
\text { Pneumonia. } & \end{array}$ & $\begin{array}{l}\text { Li K, Wu J, Wu F, Guo } \\
\text { D, Chen L, Fang Z, et } \\
\text { al. (2020) }\end{array}$ & $\begin{array}{l}\text { Investigar } \\
\text { manifestações } \\
\text { clínicas e alterações } \\
\text { em tomografia } \\
\text { computadorizada de } \\
\text { pacientes pneumonia } \\
\text { grave por COVID-19. }\end{array}$ & $\begin{array}{l}83 \text { pacientes } \text { com } \\
\text { pneumonia por COVID- } \\
19 .\end{array}$ \\
\hline $\begin{array}{l}\text { Clinical } \\
\text { characteristics and } \\
\text { imaging } \\
\text { manifestations of } \\
\text { the } 2019 \text { novel } \\
\text { coronavirus disease } \\
\text { (COVID-19): A multi- } \\
\text { center study in } \\
\text { Wenzhou city, } \\
\text { Zhejiang, China. }\end{array}$ & $\begin{array}{l}\text { Yang W, Cao Q, Qin L, } \\
\text { Wang X, Cheng Z, Pan } \\
\text { A, et al. (2020) }\end{array}$ & $\begin{array}{l}\text { Descrever as } \\
\text { características } \\
\text { clínicas } \\
\text { manifestações de } \\
\text { imagem de pacientes } \\
\text { hospitalizados com } \\
\text { infecção confirmada } \\
\text { por COVID-19 em } \\
\text { Wenzhou, Zhejiang, } \\
\text { China. }\end{array}$ & $\begin{array}{l}149 \text { pacientes com } \\
\text { diagnóstico de COVID- } \\
19 .\end{array}$ \\
\hline $\begin{array}{l}\text { Clinical findings in a } \\
\text { group of patients } \\
\text { infected with the } \\
2019 \text { novel } \\
\text { coronavirus (SARS- } \\
\text { Cov-2) outside of } \\
\text { Wuhan, China: } \\
\text { retrospective case } \\
\text { series. }\end{array}$ & $\begin{array}{l}\text { Xu XW, Wu XX, Jiang } \\
\text { XG, Xu KJ, Ying LJ, Ma } \\
\text { CL, et al. (2020) }\end{array}$ & 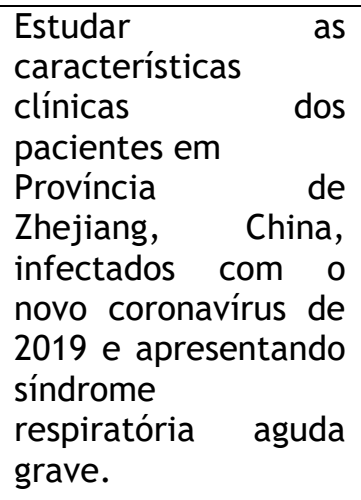 & $\begin{array}{l}62 \text { pacientes internados } \\
\text { em um hospital com } \\
\text { diagnóstico laboratorial } \\
\text { confirmado para } \\
\text { infecção por SARS-Cov- } \\
2 \text {. }\end{array}$ \\
\hline $\begin{array}{l}\text { Clinical } \\
\text { characteristics of } \\
\text { novel coronavirus } \\
\text { cases in tertiary } \\
\text { hospitals in Hubei } \\
\text { Province. }\end{array}$ & $\begin{array}{l}\text { Liu K, Fang YY, Deng Y, } \\
\text { Liu W, Wang MF, Ma JP, } \\
\text { et al. (2020) }\end{array}$ & $\begin{array}{lr}\text { Investigar a história } \\
\text { epidemiológica } \\
\text { analisar } & \text { e } \\
\text { características } & \text { as } \\
\text { clínicas, regimes de } \\
\text { tratamento r e } \\
\text { prognóstico de } \\
\text { pacientes infectados } \\
\text { com o novo } \\
\text { coronavírus. }\end{array}$ & $\begin{array}{l}\text { Dados clínicos de } 137 \\
\text { pacientes infectados } \\
\text { com o novo coronavírus. }\end{array}$ \\
\hline $\begin{array}{l}\text { Clinical } \\
\text { characteristics and } \\
\text { therapeutic } \\
\text { procedure for four } \\
\text { cases with } 2019 \\
\text { novel coronavirus } \\
\text { pneumonia receiving } \\
\text { combined Chinese } \\
\text { and Western } \\
\text { medicine treatment. }\end{array}$ & $\begin{array}{l}\text { Wang Z, Chen X, Lu Y, } \\
\text { Chen F, Zhang W. } \\
(2020)\end{array}$ & $\begin{array}{lr}\begin{array}{l}\text { Relatar } \\
\text { características }\end{array} & \text { as } \\
\text { clínicas } & \\
\text { procedimento } & \\
\text { terapêutico } & \\
\text { pacientes com } & \text { para } \\
\text { nCoV. } & \end{array}$ & $\begin{array}{l}\text { Quatro pacientes com } \\
\text { diagnóstico do novo } \\
\text { coronavírus } \\
\text { apresentando } \\
\text { pneumonia leve ou } \\
\text { grave admitidos em um } \\
\text { Centro Clínico de Saúde } \\
\text { Pública de Xangai. }\end{array}$ \\
\hline
\end{tabular}


Conflito de interesses: Os autores declaram não haver conflito de interesses.

\section{Participação dos autores:}

- Concepção: Dantas TP, Aguiar CAS, Rodrigues VRT, Silva RRG, Silva MIC, Sampaio LRL, Pinheiro WR.

- Desenvolvimento: Dantas TP, Aguiar CAS, Rodrigues VRT, Silva RRG, Silva MIC, Sampaio LRL, Pinheiro WR.

- Redação e revisão: Dantas TP, Aguiar CAS, Rodrigues VRT, Silva RRG, Silva MIC, Sampaio LRL, Pinheiro WR.

Como citar este artigo: Dantas TP, Aguiar CAS, Rodrigues VRT, Silva RRG, Silva MIC, Sampaio LRL, et al. Diagnósticos de enfermagem para pacientes com COVID-19. J Health NPEPS. 2020; 5(1):396-416.

Submissão (Fast Track COVID-19): 27/05/2020

Aceito (Fast Track COVID-19): 12/06/2020

Publicado (Fast Track COVID-19): 13/06/2020 